\title{
Dor dentária em usuários de Substâncias Psicoativas dos CAPS AD de Vitória, Vila Velha e Serra, ES, Brasil
}

\author{
Dental pain among psychoactive substance users in CAPS AD in \\ Vitoria, Vila Velha and Serra, ES, Brazil
}

Bruna Venturin Lorencini (https://orcid.org/0000-0001-6015-7016) ${ }^{1}$

Bruna Costa Bissoli (https://orcid.org/0000-0002-4004-8362) ${ }^{1}$

Jeremias Campos Simões (https://orcid.org/0000-0002-3970-0819) ${ }^{1}$

Maria Helena Monteiro de Barros Miotto (https://orcid.org/0000-0002-3227-7608) ${ }^{1}$

Marluce Mechelli de Siqueira (https://orcid.org/0000-0002-6706-5015) ${ }^{1}$

Eliana Zandonade (https://orcid.org/0000-0001-5160-3280) ${ }^{1}$

${ }^{1}$ Programa de PósGraduação em Saúde Coletiva, Universidade Federal do Espírito Santo (UFES). Av. Marechal Campos 1468, Bonfim. 29047-105 Vitória ES Brasil.brunalorencini1@ hotmail.com

\begin{abstract}
Toothache is a public health problem that causes great inconvenience to psychoactive substances users. The objective was to verify the prevalence of dental pain and its associations among psychoactive substances users from Alcohol and Drug Psychosocial Care Centers (CAPS AD) in Vitoria, Vila Velha and Serra, Espirito Santo, Brazil. A transversal study was conducted with 280 participants between June 2015 and February 2016, using five scripts: one for socio-demographic data and health perception; another for oral health; the Oral Health Impact Profile; the Alcohol Smoking and Substance Involvement Screening Test and the World Health Organization Quality of Life Test. Data were organized in frequency tables and analyzed with the SPSS 20 statistical package. Comparisons were made with Fisher's test and the Odds Ratio (OR) was used to check the strength of the association between the variables. The prevalence of pain in the population studied was $59.3 \%$, and individuals whose quality of life was impacted due to their oral conditions were 2.2 times more likely to report toothache in the last 6 months. The population studied showed a high prevalence of dental pain and the study indicates that dental pain interferes in the quality of life of psychoactive substances users who are treated at CAPS AD services in these three cities.
\end{abstract}

Key words Toothache, Oral health, Drug users.
Resumo A dor dentária é um problema de saúde pública que causa grande incômodo aos usuários de substâncias psicoativas. Objetivou-se determinar a prevalência de dor dentária e suas associações em usuários de substâncias psicoativas dos CAPS AD dos municípios de Vitória, Vila Velha e Serra, ES, Brasil. Um estudo transversal foi realizado com 280 participantes, entre junho de 2015 e fevereiro de 2016, por meio de cinco roteiros, um para os dados sociodemográficos e percepção de saúde, outro para saúde bucal, o Oral Health Impact Profile, o Alcohol Smoking and Substance Involvement Screening Test e o World Health Organization Quality of Life. Os dados foram organizados em tabelas de frequência, analisados com o pacote estatístico SPSS 20, as comparações foram feitas com o teste de Fisher e Odds Ratio (OR) foi utilizado para verificar a força da associação entre as variáveis. A prevalência de dor na população estudada foi de 59,3\% e os indivíduos que apresentaram impacto das condições bucais na qualidade de vida, tiveram 2,2 vezes mais chances de relatar dor de dente nos últimos 6 meses. A população estudada apresentou alta prevalência de dor dentária, o estudo aponta que a dor dentária interfere na qualidade de vida dos usuários de substâncias psicoativas que utilizam os serviços dos CAPS AD dos três municípios.

Palavras-chave Dor de dente, Saúde bucal, Usuários de drogas. 


\section{Introdução}

A dor dentária é uma sensação experimentada por pessoas de todas as idades, sendo considerada um importante e relevante problema de saúde pública ${ }^{1}$. Colocada como a consequência mais comum das doenças e comprometimentos bucais, tem impacto direto na qualidade de vida dos indivíduos por ela acometidos. Sua associação com fatores socioeconômicos tem se mostrado cada vez mais evidente ${ }^{2,3}$.

A saúde bucal da população brasileira é fortemente influenciada pela desigualdade social, os principais fatores socioeconômicos utilizados para indicar e classificar a condição socioeconômica nos estudos tem sido a renda per capta, escolaridade dos pais ou da mãe e o tipo de escola (pública ou privada) ${ }^{4}$.

O uso adequado dos serviços de saúde disponíveis é um fator determinante das condições de saúde da população'. Mesmo a saúde bucal sendo reconhecida como essencial, muitos brasileiros não têm acesso a esse serviço de saúde, em decorrência disso, a população convive com os problemas bucais e suas consequências, como a dor de dente, em seu cotidiano. A grande parcela da população com baixas condições socioeconômicas não encontra nos serviços públicos de saúde a assistência adequada à resolubilidade dos problemas bucais, enfrentando rotineiramente a dor de dente e suas consequências ${ }^{5}$.

A dor dentária tem se tornado objeto de muitos estudos epidemiológicos, preocupados não apenas com sua origem, mas, principalmente com os fatores individuais associados ao contexto, assim como o impacto na vida das pessoas. Os principais fatores relacionados são os socioeconômicos, demográficos, familiares, comportamentais, psicológicos e culturais ${ }^{4}$. O público alvo desses estudos tem se concentrado em crianças e adolescentes ${ }^{2,6-8}$, pouco se estuda sobre as consequências bucais do uso de substâncias psicoativas (SPAs), em especial as ilícitas9.

A avaliação da dor dentária em usuários de SPAs foi encontrada em poucos estudos internacionais $^{10-17}$. Grande parte desses estudos mostram que a dor dentária tem causado relevante incômodo a esses indivíduos, estando entre os principais problemas bucais apresentados por essa população.

Entre as drogas ilícitas utilizadas pela população brasileira, o crack é a que causa maior dependência e piores consequências para a saúde do usuário. Pouco se sabe sobre os padrões de uso e característica dos usuários dessa substância no Brasil, o conhecimento dessas características se torna importante no serviço de saúde, permitindo fundamentar melhores propostas de tratamento e otimizar recursos disponíveis ${ }^{18,19}$.

Nos últimos anos, o governo brasileiro tem objetivado melhorar a Atenção Primária à Saúde ofertada pelo Sistema Único de Saúde, através da Estratégia Saúde da Família (ESF). O Ministério da Saúde incentivou a substituição do modelo de prestação de serviços públicos odontológicos, curativos e direcionados a uma pequena parcela da população, de forma a ser ofertado a todos, por meio da inclusão das Equipes de Saúde Bucal (ESB) na $\mathrm{ESF}^{20}$. Devido às diretrizes organizacionais, a atenção aos problemas odontológicos apresentados pelos usuários de substâncias psicoativas seria facilitada nas Unidades de Saúde da Família, evitando a evolução para dor.

Os Centros de Atenção Psicossocial Álcool e Drogas (CAPS AD) devem executar suas funções ao mesmo tempo que atuam fazendo uma ligação entre o paciente e a sociedade, estando também articulado a outros serviços, como Unidades Básicas de Saúde, Estratégia Saúde da Família, equipes de saúde mental e hospitais ${ }^{21}$. No entanto, os profissionais de odontologia não fazem parte da equipe atuante nos CAPS $\mathrm{AD}^{22}$, dificultando a referência aos serviços para resolução de problemas bucais, consequentemente, a evolução resulta em dor.

Diante do exposto, este estudo teve como objetivo, identificar a prevalência da dor de origem dentária em usuários de substâncias psicoativas, avaliar a associação da dor dentária com as condições sociodemográficas, a qualidade de vida, impacto da saúde bucal na qualidade de vida e com o consumo de substâncias psicoativas pelos indivíduos assistidos nos Centros de Atenção Psicossocial Álcool e Drogas dos municípios de Vitória, Vila Velha e Serra, ES.

\section{Metodologia}

O estudo realizado foi do tipo analítico, de corte transversal, realizado nos CAPS AD dos municípios de Vitória, Vila Velha e Serra, ES, no período de junho de 2015 a fevereiro de 2016.

Este estudo teve aprovação do Comitê de Ética em Pesquisa do Centro de Ciências da Saúde da Universidade Federal do Espírito Santo segundo a resolução $n^{\circ} 466 / 12$ do Conselho Nacional de Saúde, em 27 de maio de 2015.

Os sujeitos do estudo foram os indivíduos que estavam em acompanhamento devido ao 
uso de substâncias psicoativas. Foram incluídos no estudo os indivíduos com 18 anos ou mais, e excluídos os que apresentavam comorbidades psiquiátricas ou desvios comportamentais que impedissem a execução adequada da pesquisa.

Foi realizado o cálculo amostral no programa estatístico Bioestat, versão 3.1, considerando a população de 1.000 indivíduos, prevalência de $50 \%$, nível de confiança de $95 \%$ e erro amostral de $5 \%$, chegou-se a um número mínimo para a amostra de 278 sujeitos divididos entre os três CAPS AD.

A seleção da amostra se deu por conveniência, porém, buscou-se uma melhor representatividade dos três Centros com o uso de um cronograma de visitação por parte dos pesquisadores, de forma que cada CAPS $\mathrm{AD}$ fosse visitado em dias e horários pré-estabelecidos, garantindo a todos, igual probabilidade de participar. Todos os indivíduos presentes eram convidados a participar do estudo, esse convite acontecia no início das atividades em grupos desenvolvidas pelos CAPS AD, nas salas de espera para consultas ou por abordagem individual, aos que não se encontravam presentes nos grupos no momento da abordagem coletiva.

A técnica de coleta utilizada foi de entrevista com registro em formulário, realizada por três pesquisadores. Foram utilizados cinco roteiros para coleta dos dados, um referente a informações sociodemográficas e um sobre informações de saúde bucal, necessidade e acesso a tratamento odontológico auto relatado, desenvolvidos pelos pesquisadores com base na literatura consultada, além dos questionários OHIP-14, WHOQOL e ASSIST, já validados para a realização de pesquisas epidemiológicas no Brasil ${ }^{23-25}$.

Os indivíduos que aceitavam participar do estudo eram direcionados de forma individualizada para uma sala onde eram aplicados os cinco questionários, com um tempo de aplicação de aproximadamente 30 a 40 minutos. Permaneciam na sala apenas o participante e o pesquisador.

A coleta dos dados foi estruturada por meio de um estudo preliminar realizado pelos pesquisadores em 2014 com aprovação do Comitê de Ética em Pesquisa envolvendo seres humanos (CEP) da UFES, em 27 de agosto do mesmo ano. O estudo-piloto teve como população-alvo internos de uma Comunidade Terapêutica do município de Vitória, ES, e teve como objetivo, avaliar o perfil sociodemográfico dos usuários daquele serviço e descrever as substâncias psicoativas mais utilizadas pela população estudada nos três meses anteriores à pesquisa.
O questionário OHIP-14 foi utilizado para coletar os dados sobre impacto da saúde bucal na qualidade de vida. As respostas foram codificadas por meio de uma escala de Lickert, com cinco opções para cada problema apresentado nos últimos 6 meses: sempre, quase sempre, às vezes, poucas vezes e nunca. Foi feita uma avaliação dicotômica das respostas obtidas, onde "às vezes" "poucas vezes" e "nunca" indicam que não houve impacto e "sempre" e "quase sempre" indicam impacto.

A qualidade de vida geral foi medida por meio das duas primeiras questões do instrumento WHOQOL-bref, apesar do roteiro ser composto por 26 questões. Essas variáveis foram utilizadas de forma dicotômica. A primeira relacionada a qualidade de vida geral, tendo como opções de resposta "muito ruim", "ruim", "nem ruim nem boa", "boa" e "muito boa", a segunda relacionada a satisfação com a saúde, com as opções "muito insatisfeito", "insatisfeito", "nem satisfeito nem insatisfeito", "satisfeito", "muito satisfeito".

O questionário ASSIST foi utilizado para mensurar o uso das substâncias. O ASSIST classifica o uso baixo, moderado e alto risco para dependência. Trata-se de um questionário estruturado, com oito questões referentes ao uso de tabaco, álcool, maconha, cocaína, estimulantes, inalantes, sedativos, alucinógenos e opióides, relacionadas ao uso na vida, a frequência dos últimos três meses, sobre o desejo ou urgência em usar a droga, dificuldade em controlar, diminuir ou interromper o uso, problemas relacionados ao uso, não cumprimento das obrigações e compromissos devido ao uso, preocupação com o uso por pessoas próximas e o uso de droga injetável. As opções possuem uma pontuação, se o resultado da soma ficar entre 0 e 10 para o álcool e de 0 a 3 para as demais substâncias indica baixo risco, de 11 a 26 para o álcool e 4 a 26 para as demais, indica risco moderado e acima de 26 indica alto risco de dependência.

A variável dependente desse estudo, dor de origem dentária nos últimos seis meses, foi extraída do questionário sobre saúde bucal, já as variáveis independentes foram extraídas de quatro instrumentos, variáveis sociodemográficas (sexo, faixa etária, cor, estado civil, escolaridade do usuário, renda familiar, fonte de renda, bolsa do governo, moradia), relacionadas ao estado de saúde e qualidade de vida (estado de saúde nos últimos 30 dias, satisfação com a saúde e auto avaliação da qualidade de vida; obtidos por meio das questões 1 e 2 do questionário WHOQOL), consumo de substâncias (maconha, cocaína, crack, crack/ 
cocaína, álcool e tabaco; obtidas com o questionário ASSIST), impacto das condições bucais na qualidade de vida, extraída do OHIP-14.

Os dados foram organizados em tabelas de frequência com número e percentual para cada um dos itens. A comparação do uso e frequência do uso de drogas e qualidade de vida com os fatores demográficos foi testada através do teste de Fisher. O Odds Ratio (OR) foi utilizado para verificar a força da associação. O nível de significância adotado foi de 5\%. A análise foi realizada com o pacote estatístico IBM SPSS 20.

\section{Resultados}

Participaram do estudo, 280 sujeitos distribuídos proporcionalmente entre os CAPS AD de Vitória $(\mathrm{n}=95)$, Vila Velha $(\mathrm{n}=91)$ e Serra $(\mathrm{n}$ =94). A amostra foi composta em sua maioria, por indivíduos do sexo masculino, 74,3\% ( $\mathrm{n}=$ 208), as idades variaram de 19 a 84 anos, com maior concentração nas faixas etárias abaixo dos 51 anos. Quanto ao auto relato da cor, 60\% ( $\mathrm{n}=$ 168) da amostra se considera parda, $21,1 \%(\mathrm{n}=$ 59) branca e $18,2 \%(n=51)$ negra. Com relação ao estado civil, $46,1 \%(\mathrm{n}=129)$ dos indivíduos eram solteiros; apenas $28,2 \%(n=79)$ da amostra completou o ensino médio (Tabela 1).

Na Tabela 2 é possível observar os dados de percepção do estado de saúde da população estudada. Quando questionados sobre o estado de saúde, apenas $6,8 \%(\mathrm{n}=19)$ dos participantes consideraram seu estado de saúde excelente nos últimos 30 dias e $32,9 \%(n=92)$ consideraram ruim, o restante ficou dividido entre bom e satisfatório. Quanto à satisfação com a saúde, apenas $8,6 \%(\mathrm{n}=24)$ dos participantes estavam muito satisfeitos. Quanto à qualidade de vida, apenas $5,4 \%(\mathrm{n}=15)$ avaliam sua qualidade de vida como muito boa, $27,9 \%(\mathrm{n}=78)$ a consideraram ruim.

Apesar da alta de prevalência de dor dentária na população estudada $(59,3 \%)$, não foram encontradas associações estatisticamente significantes quando relacionada com as variáveis sociodemográficas (Tabela 3 ).

A dor de origem dentária nos últimos seis meses associada com baixo e alto risco para dependência em tabaco, álcool, maconha, cocaína, crack, crack e cocaína associados, não apresentou significância estatística, assim como sua associação com qualidade de vida. Houve associação apenas com o OHIP, relacionando a ocorrência de impacto das condições bucais na qualidade
Tabela 1. Dados sociodemográficos de usuários dos Centros de Atenção Psicossocial Álcool e Drogas de Vitória, Vila Velha e Serra, ES.

\begin{tabular}{|c|c|c|}
\hline Característica & $\mathbf{N}$ & $\%$ \\
\hline \multicolumn{3}{|l|}{ Sexo } \\
\hline Feminino & 72 & 25,7 \\
\hline Masculino & 208 & 74,3 \\
\hline \multicolumn{3}{|l|}{ Faixa etária } \\
\hline Até 32 anos & 73 & 26,1 \\
\hline $33-41$ anos & 70 & 25,0 \\
\hline $42-51$ anos & 75 & 26,8 \\
\hline 52 anos ou mais & 62 & 22,1 \\
\hline \multicolumn{3}{|l|}{ Raça/Cor } \\
\hline Branco & 59 & 21,1 \\
\hline Negro & 51 & 18,2 \\
\hline Amarelo & 2 & 0,7 \\
\hline Pardo & 168 & 60,0 \\
\hline \multicolumn{3}{|l|}{ Estado civil } \\
\hline Solteiro & 129 & 46,1 \\
\hline Casado & 49 & 17,5 \\
\hline Comunhão estável & 32 & 11,4 \\
\hline Separado & 62 & 22,1 \\
\hline Viúvo & 8 & 2,9 \\
\hline \multicolumn{3}{|l|}{ Escolaridade do usuário } \\
\hline Até 3 anos de estudo & 35 & 12,5 \\
\hline Ensino fundamental incompleto & 98 & 35,0 \\
\hline $\begin{array}{l}\text { Ensino fundamental completo e } \\
\text { médio incompleto }\end{array}$ & 68 & 24,3 \\
\hline Ensino médio completo e acima & 79 & 28,2 \\
\hline Total & 280 & 100,0 \\
\hline
\end{tabular}

Tabela 2. Percepção sobre condição de saúde de usuários dos Centros de Atenção Psicossocial Álcool e Drogas de Vitória, Vila Velha e Serra, ES.

\begin{tabular}{lrr}
\hline \multicolumn{1}{c}{ Característica } & N & \% \\
\hline Como considera seu estado de saúde nos & \\
últimos 30 dias & & \\
$\quad$ Excelente & 19 & 6,8 \\
Muito bom & 8 & 2,9 \\
Bom & 80 & 28,5 \\
Satisfatório & 81 & 28,9 \\
Ruim & 92 & 32,9 \\
Quão satisfeito está com a sua saúde & & \\
Muito insatisfeito & 35 & 12,5 \\
Insatisfeito & 79 & 28,2 \\
Nem satisfeito, nem insatisfeito & 79 & 28,2 \\
Satisfeito & 63 & 22,5 \\
Muito satisfeito & 24 & 8,6 \\
Como avalia sua qualidade de vida & & \\
Muito ruim & 30 & 10,7 \\
Ruim & 78 & 27,9 \\
Nem ruim, nem boa & 88 & 31,4 \\
Boa & 69 & 24.6 \\
Muito boa & 15 & 5,4 \\
\hline
\end{tabular}


Tabela 3. Dor dentária, segundo dados sociodemográficos de usuários dos Centros de Atenção Psicossocial Álcool e Drogas de Vitória, Vila Velha e Serra, ES.

\begin{tabular}{|c|c|c|c|c|c|c|}
\hline \multirow{2}{*}{ Variável } & \multicolumn{2}{|c|}{ Com dor } & \multicolumn{2}{|c|}{ Sem dor } & \multirow{2}{*}{ Sig. } & \multirow{2}{*}{ OR } \\
\hline & No & $\%$ & No & $\%$ & & \\
\hline \multicolumn{7}{|l|}{ Sexo } \\
\hline Masculino & 122 & 58,7 & 86 & 41,3 & 0,412 & 1,107 \\
\hline Feminino & 44 & 61,1 & 28 & 38,9 & & $0,640-1,916$ \\
\hline \multicolumn{7}{|l|}{ Faixa etária } \\
\hline Até 41 anos & 81 & 56,6 & 62 & 43,4 & 0,213 & 1,252 \\
\hline 42 anos ou mais & 85 & 62,0 & 52 & 38,0 & & $0,776-2,016$ \\
\hline \multicolumn{7}{|l|}{ Cor } \\
\hline Branco & 34 & 57,6 & 25 & 42,4 & 0,441 & 1,091 \\
\hline Não branco & 132 & 59,7 & 89 & 40,3 & & $0,609-1,953$ \\
\hline \multicolumn{7}{|l|}{ Estado civil } \\
\hline Casado/União estável & 59 & 61,7 & 31 & 38,3 & 0,347 & 1,154 \\
\hline Solteiro/separado/viúvo & 116 & 58,3 & 83 & 41,7 & & $0,680-1,959$ \\
\hline \multicolumn{7}{|l|}{ Anos estudo usuário } \\
\hline Até 8 anos & 100 & 58,1 & 72 & 41,9 & 0,357 & 1,131 \\
\hline 9 anos ou mais & 66 & 61,1 & 42 & 38,9 & & $0,692-1,848$ \\
\hline \multicolumn{7}{|l|}{ Anos estudo chefe } \\
\hline Até 8 anos & 93 & 58,9 & 65 & 41,1 & 0,272 & 1,218 \\
\hline 9 anos ou mais & 61 & 63,5 & 35 & 36,5 & & $0,723-2,053$ \\
\hline \multicolumn{7}{|l|}{ Renda } \\
\hline Até 1 salário mínimo & 93 & 58,9 & 65 & 41,1 & 0,334 & 1,148 \\
\hline Mais de 1 salário mínimo & 61 & 63,5 & 35 & 36,5 & & $0,705-1,868$ \\
\hline \multicolumn{7}{|l|}{ Fonte de renda ${ }^{\star}$} \\
\hline Sem fonte de renda & 46 & 61,3 & 29 & 38,7 & 0,389 & 1,124 \\
\hline Com fonte de renda & 120 & 58,5 & 85 & 41,5 & & $0,654-1,931$ \\
\hline \multicolumn{7}{|l|}{ Bolsa do governo } \\
\hline Sim & 21 & 65,6 & 11 & 34,4 & 0,282 & 1,356 \\
\hline Não & 145 & 58,5 & 103 & 41,5 & & $0,627-2,935$ \\
\hline \multicolumn{7}{|l|}{ Moradia ${ }^{* *}$} \\
\hline Sem moradia & 35 & 60,3 & 23 & 39,7 & 0,489 & 1,057 \\
\hline Com moradia & 131 & 57,3 & 91 & 42,7 & & $0,586-1,907$ \\
\hline
\end{tabular}

de vida com dor de origem dentária. Indivíduos com impacto apresentaram 2,2 vezes mais chances de relatar dor de dente nos últimos 6 meses, OR $=2,2$ (Tabela 4$)$.

\section{Discussão}

Esse estudo avaliou a dor de origem dentária nos usuários de substâncias psicoativas atendidos nos CAPS AD de Vitória, Vila Velha e Serra, ES, e sua relação com a qualidade de vida e uso de substâncias. $\mathrm{Na}$ amostra de 280 participantes, houve uma prevalência de indivíduos do sexo masculino $(74,3 \%)$, com idade abaixo dos 52 anos, de cor parda, solteiros, com ensino médio incompleto. Esses dados estão de acordo com estudos realizados em Goiás e em Campina Grande ${ }^{26,27}$.

A renda familiar no grupo esteve abaixo de dois salários mínimos, e 26,8\% dos participantes não recebem nenhum tipo de ajuda, benefício ou trabalham de forma regular. Uma grande parcela dos indivíduos, $(62,8 \%)$ relataram viver com ajuda da família ou algum benefício.

Grande parte da população estudada relatou ter apresentado dor dentária nos últimos 6 meses. Estes resultados corroboram com a literatura, um estudo realizado nos Estados Unidos, encontrou que os usuários de metanfetaminas tem 3,5 vezes mais chances de apresentarem dor 
Tabela 4. Dor dentária, segundo dados de consumo de drogas e qualidade de vida de usuários dos Centros de Atenção Psicossocial Álcool e Drogas de Vitória, Vila Velha e Serra, ES.

\begin{tabular}{|c|c|c|c|c|c|c|}
\hline \multirow{2}{*}{ Variável } & \multicolumn{2}{|c|}{ Com dor } & \multicolumn{2}{|c|}{ Sem dor } & \multirow{2}{*}{ Sig. } & \multirow{2}{*}{ OR } \\
\hline & No & $\%$ & No & $\%$ & & \\
\hline \multicolumn{7}{|l|}{ Consumo de Tabaco } \\
\hline Baixo risco & 17 & 60,7 & 11 & 39,3 & \multirow[t]{2}{*}{0,521} & 1,068 \\
\hline Risco/Dependente & 136 & 59,1 & 94 & 40,9 & & $0,479-2,384$ \\
\hline \multicolumn{7}{|l|}{ Consumo de Álcool } \\
\hline Baixo risco & 41 & 58,6 & 29 & 41,4 & \multirow[t]{2}{*}{0,493} & 1,044 \\
\hline Risco/Dependente & 124 & 59,6 & 84 & 40,4 & & $0,602-1,812$ \\
\hline \multicolumn{7}{|l|}{ Consumo de Maconha } \\
\hline Baixo risco & 64 & 63,4 & 37 & 36,6 & \multirow[t]{2}{*}{0,082} & 1,560 \\
\hline Risco/Dependente & 51 & 52,6 & 46 & 47,4 & & $0,884-2,753$ \\
\hline \multicolumn{7}{|l|}{ Consumo de Cocaína } \\
\hline Baixo risco & 53 & 65,4 & 28 & 34,6 & \multirow[t]{2}{*}{0,073} & 1,627 \\
\hline Risco/Dependente & 57 & 53,8 & 49 & 46,2 & & $0,896-2,954$ \\
\hline \multicolumn{7}{|l|}{ Consumo de Crack } \\
\hline Baixo risco & 20 & 69,0 & 9 & 31,0 & \multirow[t]{2}{*}{0,200} & 1,576 \\
\hline Risco/Dependente & 86 & 58,5 & 61 & 41,5 & & $0,672-3,697$ \\
\hline \multicolumn{7}{|l|}{ Consumo de Crack e Cocaína } \\
\hline Baixo risco & 24 & 66,7 & 12 & 33,3 & \multirow[t]{2}{*}{0,219} & 1,453 \\
\hline Risco/Dependente & 95 & 57,9 & 69 & 42,1 & & $0,680-3,103$ \\
\hline \multicolumn{7}{|l|}{ Qualidade de Vida } \\
\hline Muito ruim/Ruim/Regular & 114 & 58,2 & 82 & 41,8 & \multirow[t]{2}{*}{0,327} & 1,168 \\
\hline Boa/Muito boa & 52 & 61,9 & 32 & 38,1 & & $0,692-1,972$ \\
\hline \multicolumn{7}{|l|}{ OHIP } \\
\hline Sem impacto & 34 & 39,5 & 52 & 60,5 & \multirow[t]{2}{*}{0,000} & 2,257 \\
\hline Com impacto & 132 & 68,0 & 62 & 32,0 & & $1,923-5,525$ \\
\hline
\end{tabular}

de origem dentária quando comparados aos não usuários, e entre os usuários, indivíduos que fazem uso pesado (16-30 vezes nos últimos 30 dias) relataram mais dor de dente ${ }^{10}$.

Um estudo realizado nos Estados Unidos com usuários de álcool, encontrou a dor de dente como o principal motivo para os participantes irem ao cirurgião dentista ${ }^{12}$, outro estudo comparando fumantes e não fumantes, encontrou que os fumantes têm 1,5 vezes mais chances de relatar dor de dente ${ }^{16}$. Já um outro, mostrou a dor de dente como o principal problema apresentado no último ano por usuários de diversas substâncias, quando comparados a não usuários ${ }^{11}$.

A prevalência de dor dentária nos últimos seis meses na população estudada foi de 59,3\%. Não foram encontrados estudos realizados no Brasil, avaliando dor de dente em usuários de drogas; estudos realizados no Espirito Santo, na região sudeste do Brasil e no Brasil, com a população geral, apontam prevalência de dor para os últimos seis meses, inferior a encontrada nesse estudo. Um estudo realizado em Venda Nova do
Imigrante-ES, encontrou prevalência de dor de $43 \%{ }^{28}$, em outro, realizado em um município do norte do estado, a dor dentária apresentou prevalência de $46,7 \%{ }^{29}$. Percentual ainda menor apresenta a prevalência de dor dentária encontrada no último levantamento nacional, onde, para a faixa etária de 35 a 44 anos, a região sudeste do país apresentou $27,5 \%$ e a média nacional foi de $30,8 \%{ }^{30}$.

Os indivíduos que apresentaram impacto das condições bucais na qualidade de vida tiveram 2,2 vezes mais chances de relatar dor de dente nos últimos seis meses. A população estudada se declarou insatisfeita com seu estado de saúde e qualidade de vida, no entanto, não foram encontradas associações entre a dor de origem dentária e a qualidade de vida geral, especificamente, embora muitos estudos afirmem que as condições bucais refletem diretamente na qualidade de vida ${ }^{1-3,9}$.

$O$ instrumento WHOQOL tem sensibilidade para a qualidade de vida, enquanto o OHIP-14 mensura exclusivamente o impacto das condições bucais na qualidade de vida. Torna-se neces- 
sária a realização de novos estudos para melhor entender o significado de dor de dente para a população estudada, quando questionada sobre qualidade de vida de forma geral.

Quando se avaliou a possível associação entre a variável de estudo e o uso de cada substância não foram encontradas associações estatisticamente significantes, esse resultado vai ao encontro de um estudo realizado nos Estados Unidos, com 650 usuários de diversas substâncias, onde não foram encontradas associações significantes entre o uso da cada substância com a dor de dente, podendo ser resultado do uso associado de várias substâncias ${ }^{15}$. Se torna difícil relacionar as alterações bucais encontradas nos dependentes químicos com um tipo específico de substância, isso se deve principalmente ao fato dos indivíduos fazerem uso de várias substâncias ao mesmo tempo'.

A baixa renda da população estudada, a preocupação com o estado de saúde, insatisfação com a saúde e qualidade de vida e a alta prevalência de dor dentária, indicam que os usuários de substâncias psicoativas estudados dependem do sistema público de saúde para atender às suas necessidades, especialmente as de saúde bucal. Para que seja dada completa atenção às necessidades dos usuários, os CAPS AD devem atuar de forma conjunta com as UBS e equipes da ESF, como preconiza o SUS e a rede de atenção em saúde mental, porém, estudos realizados em diferentes regiões do Brasil, com equipes da ESF, apontam que os profissionais se encontram despreparados e pouco qualificados para o atendimento a esse público $^{31,32}$.

Um estudo realizado com o objetivo de avaliar o atendimento aos usuários de SPAs no contexto da rede de atenção em saúde mental, a partir de uma ESF, encontrou vários problemas apontados pelos trabalhadores, entre eles a dificuldade de proporcionar acesso a serviço especializado e quando essa dificuldade era superada, faltavam recursos territoriais para dar continuidade ao tratamento. Os trabalhadores acreditam que essa situação é reflexo da ausência de impacto das políticas de saúde mental voltadas para os usuários de substâncias na atenção primária em saúde, especialmente na ESF. Além disso, ficou evidente a desconexão do serviço com outros pontos de atenção à saúde mental no município, evidenciando a precariedade de ofertas de ações voltadas para esses indivíduos no território ${ }^{33}$.

Outro estudo aponta que apesar da gravidade dos problemas de saúde bucal entre os usuários de substâncias e os efeitos positivos dos cuidados bucais no tratamento do vício, a prestação de serviços odontológicos para esses pacientes enfrenta desafios como: dificuldade de acesso, falta de protocolos de avaliação para detectar patologias orais comuns a essa população, falta de interação entre profissionais e setores que prestam cuidados de saúde bucal e geral a esses indivíduos, a falta de políticas de acesso aos serviços odontológicos e a falta de conhecimento e interesse dos cirurgiões-dentistas nessa área. Os programas de saúde bucal devem ser integrados aos de saúde geral já existentes para esse público ${ }^{34}$.

É importante pensar na inclusão de profissionais de odontologia qualificados para atuação nos CAPS AD, compondo uma equipe complementar à equipe de saúde mental que já atua nesses espaços, funcionando como um facilitador para a atenção integral aos problemas bucais dos usuários de substâncias psicoativas, atuando de forma conjunta com os profissionais da ESF.

Considerando que a população estudada tem alta prevalência de utilização dos serviços odontológicos e de dor dentária, se faz necessária a ampliação e organização de programas de qualificação ofertados para os cirurgiões-dentistas que atuam na Atenção Primária dos municípios estudados, assim como, melhorar as condições de trabalho desses profissionais para que deem continuidade ao tratamento iniciado no CAPS AD, propiciando a resolução dos problemas bucais desses indivíduos no próprio território a que pertencem, evitando a evolução para dor dentária.

Se faz necessário o desenvolvimento de estudos que permitam a comparação desse público com uma população de indivíduos não usuários de substâncias, para que melhores associações sejam feitas entre o uso de drogas e a ocorrência de dor dentária. Estudos que avaliem de forma mais aprofundada a associação entre dor dentária e variáveis sociodemográficas em usuários de SPAs também se fazem necessários.

\section{Conclusão}

Os usuários de substâncias psicoativas atendidos nos CAPS AD dos municípios de Vitória, Vila Velha e Serra, apresentam alta prevalência de dor dentária o que indica condições bucais ruins. Indivíduos que apresentam dor de dente estão mais propensos a sofrer impactos das condições bucais na qualidade de vida.

Os profissionais envolvidos no atendimento a esse público precisam conhecer os transtornos bucais por eles enfrentados, para que seja feita a 
referência à atenção Básica ou Estratégia Saúde da Família, proporcionando um atendimento integral, já que as condições bucais interferem na qualidade de vida desses indivíduos.

Programas de qualificação profissional precisam ser desenvolvidos para tornarem os profissionais de saúde que atuam na atenção primária, aptos e seguros no atendimento clínico de usuários de substâncias psicoativas.

O cuidado em saúde a esse público, precisa ser ofertado de forma interdisciplinar, envolvendo os mais diversos profissionais, inclusive o $\mathrm{Ci}$ rurgião-Dentista, tanto na atenção primária em saúde, quanto no espaço dos CAPS AD.

\section{Colaboradores}

BV Lorencini: contribuições ao projeto e desenvolvimento da pesquisa, redação do trabalho e revisão crítica do conteúdo. BC Bissoli: contribuições ao projeto e desenvolvimento da pesquisa e aprovação final da versão a ser publicada. JC Simões: contribuições ao projeto e desenvolvimento da pesquisa e aprovação final da versão a ser publicada. E Zandonade: contribuições ao projeto, revisão crítica do conteúdo e aprovação final da versão a ser publicada. MM Siqueira: contribuições ao projeto, revisão crítica do conteúdo e aprovação final da versão a ser publicada. MHMB Miotto: contribuições ao projeto e desenvolvimento da pesquisa, revisão crítica do conteúdo e aprovação final da versão a ser publicada. 


\section{Referências}

1. Hamati F, Rocha JS, Baldani MH. Prevalência de cárie, dor e uso de serviços odontológicos por crianças em áreas com e sem Equipes de Saúde Bucal na Estratégia Saúde da Família no município de Ponta Grossa, Paraná, Brasil. Rev Bras Pesq Saude 2014;16(3):48-57.

2. Souza JGS, Martins AMEBL. Dental pain and associated factors in Brazilian preschoolers. Rev Paul Pediatr 2016;34(3):336-342.

3. Peres MA, Iser BPM, Peres KG, Malta DC, Antunes JLF. Desigualdades contextuais e individuais da prevalência de dor dentária em adultos e idosos no Brasil. Cad Saude Publica 2012;28(Supl.):114-123.

4. Freire MCM, Leles CR, Sardinha LMV, Junior MP Malta DC, Peres MA. Dor dentária e fatores associados em adolescentes brasileiros: a Pesquisa Nacional de Saúde do Escolar (PeNSE), Brasil, 2009. Cad Saude Publica 2012;28(Supl.):133-145.

5. Ferreira AAA, Piuvezam G, Werner CW, Alves MS. A dor e a perda dentária: representações sociais do cuidado à saúde bucal. Ciên Saude Colet 2006;11(1):211-218.

6. Guskuma RC, Lages VA, Hafner MB, Meireles MPMR Cypriano S, Sousa MLR, Batista MJ. Fatores associados a prevalência e intensidade de odontalgia em crianças de Municípios da Região de Campinas, São Paulo. Rev Paul Pediatr 2017;35(3):322-330.

7. Ortiz FR, Ramadan YH, González RAB, Ardenghi TM. Fatores associados com a qualidade de vida relacionada à Saúde Bucal de pré-escolares no sul do Brasil. Rev Gaúch Odontol 2016;64(3):256-262.

8. Gomes MC, Perazzo MF, Neves ET, Martins CC, Paiva SM, Garcia AFG. Oral Problems and Self-Confidence in Preschool Children. Braz Dent J 2017;28(4):523530.

9. Alves DM, Nai GA, Parizi JLS. Avaliação da ação do uso de drogas na saúde bucal de dependentes químicos. Colloquium Vitae 2013;5(1):40-58.

10. Murphy DA, Harrell L, Fintzy R, Belin TR, Gutierrrez A, Vitero SJ, Shetty V. A Comparison of Methamphetamine Users to a Matched NHANES Cohort: Propensity Score Analyses for Oral Health Care and Dental Service Need. J Behav Health Serv Res 2014;1-4.

11. Sheridan J, Aggleton M, Carson T. Dental health and access to dental treatment: a comparison of drug users and non-drug users attending community pharmacies. Br Dent J 2001;191(8):453-457.

12. Khocht A, Schleifer SJ, Janal MN, Keller S. Dental care and oral disease in alcohol-dependent persons. J Subst Abuse Treat 2009;37(2):214-218.

13. Moreno MVM, Highsmith JDR, Garcia RR, Ruiz MFS, Viñuela AC. Dental profile of a community of recovering drug addicts: Biomedical aspects. Retrospective cohort study. Med Oral Patol Oral Cir Bucal 2013;18(4):671-679.

14. Laslett AM, Dietze P, Dwyer R. The oral health of street-recruited injecting drug users: prevalence and correlates of problems. Addiction 2008;103(11):18211825 .

15. D'amore MM, Cheng DM, Kressin NR, Jones J, Samet JH, Winter M, Kim TW, Saitz R. Oral health of substance-dependent individuals Impact of specific substances. J Subst Abuse Treat 2011;41(2):179-185.
16. Riley JM, Tomar SL, Gilbert GH. Smoking and Smokeless Tobacco: Increased Risk for Oral Pain. The Journal of Pain 2004;5(4):218-225.

17. Smit DA, Naidoo S. Methamphetamine abuse: Oral symptoms and dental treatment needs. SADJ 2016;9(4): 148-152.

18. Vargens RW, Cruz MS, Santos M A. Comparação entre usuários de crack e de outras drogas em serviço ambulatorial especializado de hospital universitário. Revista Latino-americana de Enfermagem 2011;19(Spe):804-812.

19. Botti NCL, Machado JSA, Tameirão FV. Perfil sociodemográfico e padrão do uso de crack entre usuários em tratamento no Centro de Atenção Psicossocial. Estud Pesqui. Psicol 2014;14(1):290-303.

20. Stocco G, Baldani MH. O controle das consultas odontológicas dos bebês por meio da carteira de vacina: avaliação de um programa-piloto desenvolvido na Estratégia Saúde da Família em Ponta Grossa (PR, Brasil). Ciên Saude Colet 2011;16(4): 2311-2321.

21. Silveira EAA, Oliveira PP, Correio PM, Santos WJ, Rodrigues AB, Costa, DARS. O cuidado aos dependentes químicos: com a palavra profissionais de saúde de centros de atenção psicossocial em álcool e drogas. J Res Fundam Care 2016;8(2):4347-4364.

22. Falcão CAM, Santos RO, Pereira RMS, Silva TSO, Ferreira RS, Silva FWC, Sousa MP, Ferraz MAAL. Saúde bucal em dependentes químicos. Rev Interd Ciên Saúde 2015;2(3):112-121.

23. Oliveira BH, Nadanovsky P. Psychometric properties of the Brazilian version of the Oral Health Impact Profile-short form. Comm Dent Oral Epidemiol 2005;33(4):307-314

24. Fleck MPA, Louzada S, Xavier M, Chachamovich E, Vieira G, Santos L, Pinzon V. Aplicação da versão em português do instrumento de avaliação de qualidade de vida da organização mundial da saúde (WHOQOL-100) 1999. Rev Saude Pub 1999;34(2):198-205.

25. Henrique IFS, Micheli D, Lacerda RB, Lacerda LA, Formigoni MLOS. Validação da versão brasileira do teste de triagem do desenvolvimento com álcool, cigarro e outras substâncias (ASSIST). Rev Assoc Med Bras 2004;50(2):199-206.

26. Guimarães RA, Silva NL, França DDS, Del-Rios NHA, Carneiro MAS, Teles SA. Comportamentos de risco para doenças sexualmente transmissíveis em usuários de crack. Rev Latino-Am Enfermagem 2015;23(4):628634.

27. Costa SKP, Godoy GP, Gomes DQ, Pereira JV, Lins DAU. Fatores Sociodemográficos e Condições de Saúde Bucal em Droga-Dependentes. Pesq Bras Odontoped Clin Integr 2011;11(1):99-104.

28. Miotto MHMB, Silloti JCB, Barcellos LA. Dor dentária como motivo de absenteísmo em uma população de trabalhadores. Cien Saude Colet 2012;17(5):1357-1363.

29. Miotto MHMB, Barcellos LA, Lopes ZV. Dor de dente como preditor de absenteísmo em trabalhadores de uma indústria de sucos da Região Sudeste do Brasil. Cien Saude Colet 2013;18(11):3183-3190.

30. Brasil. Ministério da Saúde (MS). Secretaria de Atenção à Saúde. Secretaria de Vigilância em Saúde. SB Brasil 2010: pesquisa nacional de saúde bucal-resultados principais. Brasília: MS; 2012. 
31. Laport TJ, Costa PHA, Mota DCB, Ronzani TM. Percepções e Práticas dos Profissionais da Atenção Primária à Saúde na Abordagem sobre Drogas. Psicologia: Teoria e Pesquisa 2016;32(1)143-150.

32. Tonhom SFR, Albino Filho MA, Belotti LD, Lima JP. Atenção integral aos usuários de álcool e outras drogas no cenário da Atenção Básica. Atas - Investigação Qualitativa em Saúde 2016; 2:1098-1106.

33. Schneider JF, Roos CM, Olschowsky A, Pinho LB, Camatta MW, Wetzel C. Atendimento a usuários de drogas na perspectiva dos profissionais da Estratégia Saúde da Família. Texto Contexto Enferm 2013;22(3):654-661.

34. Shekarchizadeh H, Khami MR, Mohebbi SZ, Ekhtiari H, Virtanen JI. Oral Health of Drug Abusers. Iranian J Publ Health 2013;42(9):929-940.

Artigo apresentado em 09/04/2017

Aprovado em 22/04/2018

Versão final apresentada em 24/04/2018 